\title{
06
}

\section{Low- and High-Order Nonlinear Optical Characterization of Metal Sulfide Quantum Dots*}

\author{
(C) R.A. Ganeev
}

The Guo China-US Photonics Laboratory, Changchun Institute of Optics, Fine Mechanics and Physics, Chinese Academy of Sciences, 130033 Changchun, China

e-mail: rashid_ganeev@mail.ru

Received March 07, 2019

Revised March 07, 2019

Accepted March 22, 2019

Any new synthesized semiconductor nanoparticles or quantum dots (QDs) require to be examined under different conditions using laser pulses of variable energies, wavelengths and durations to understand the nonlinear optical mechanisms and distinguish their attractive properties for practical applications. Among various QDs, the metal sulfides took special attention due to their advanced nonlinear optical properties. Additionally, laser ablation can readily produce plasmas containing QDs that could be used for different applications. One of them is that they can be used as emitters for harmonic generation from ultrashort laser pulses. Here we review recent studies of different low- and high-order optical nonlinearities of metal sulfide QDs, such as optical limiting, two-photon, saturable and reverse saturable types of nonlinear absorption, nonlinear refraction, and generation of high-order harmonics in extreme ultraviolet range.

Key words: nonlinear optics, metal sulfide quantum dots

DOI: $10.21883 /$ OS.2019.08.48044.96-19

* Полная версия статьи опубликована в английской версии журнала. 